Ann. Biol. anim. Bioch. Biophys., I973, 13 (2), 2I5-223.

\title{
DEVELOPMENTAL CHANGES IN SERUM PROTEINS, LIPIDS AND CHOLESTEROL DURING THE COURSE OF FORCE FEEDING IN GEESE
}

\author{
K. A. YAMANI, I. F. M. MARAI and S. LOSONCSY* \\ Department of Animal Production, \\ Faculty of Agriculture at Zagazig, \\ University of Ain Shams, Egypt \\ * Geese Research Centre, \\ University of Agriculture, \\ Gödöllö, Hungary
}

\section{SUMMARY}

Ten females and ten males of each of the Rhenish, Italian and Landes breeds of geese were force fed for four weeks. Blood samples were collected from the birds at weekly intervals beginning from the week before force feeding, the total protein and its fractions, total lipid and cholesterol were determined in the faultless blood serum samples.

As regards total proteins level, the values did not show a definite trend during the experimental period. The differences between breeds was only significant at the fourth week of cramming. Specifically, the difference was highly significant between the Rhenish and the Italian and between this last breed and the Landes breed.

The percentage of albumin increased during the experimental period in the breeds of geese studied. The total globulin percentage decreased during the same period in the same breeds. The albumin to globulin ratio in the blood sera of geese was 0.78 before cramming and I.I 4 at the end of the experiment. Differences in the percentage of albumin, globulin to globulin ratio due to the breed were not significant.

Both sera lipid and cholesterol level increased during the force feeding period. The breed differences were statistically not significant before the force feeding procedure in both items. At the third and fourth weeks of force feeding, breed showed a highly significant effect of the cholestrol level.

\section{INTRODUCTION}

The blood picture of geese during the fattening period was demonstrated by many workers (Forenbacher, I969; TOURnut and MonTLAUR-FERRADOU, I969 and WYRzYKowSKI and BEILINSKI, I969). The literature is scanty concerning the 
quantitative changes in serum proteins, lipids and cholestrol during the course of force feeding in the same kind of birds (BLuM et al. I970).

The aim of the present work is to investigate the developmental changes in serum proteins, lipids and cholestrol during the course of force feeding in geese. The effect of breed on these is also studied.

\section{MATERIALS AND METHODS}

Ten females and ten males of random-bred geese of each of the Rhenish, Italian and Landes breeds were used in the study, The average body weight was about $3.5 \mathrm{~kg}$ in females and about$4.0 \mathrm{~kg}$ in males. The birds were nine weeks old and were reared and treated under similar manage rial and environmental conditions. Force feeding was practised for a period of 28 days by electric force feeding machine to obtain fatty liver (foie gras production). The average weight of maize consumed per goose was $\mathrm{I}_{7} \mathrm{~kg}$ during the force feeding period.

Blood samples were taken from the wing vein with a very sharp needle and a syringe at weekly intervals for five weeks beginning from the week before force feeding. The volume of each blood sample was five millilitres. These samples were taken from each individual at a fixed time during the day (I I a.m.). Half an hour after collecting the samples, the blood clot was separated from the wall of each tube to facilitate the separation of the serum. The serum was separated by centrifugation after keeping the samples in a water bath at $37^{\circ} \mathrm{C}$ for another half an hour. This last step and the following ones were only carried out on the successful and faultless (not haemolized) blood samples. The samples were then transferred into other labelled test tubes which were kept in the refrigerator until analyzed. The determination of the total protein and lipid in the blood serum was carried out after BALINT (I962), and the separation of the protein fraction was achieved by paper electrophoresis suggested by DitTMER and ANNo (I965). The examination of the cholestrol was done after LEvine and ZAK (I969).

The statistical analysis was carried out after SvAB (I967).

\section{RESULTS AND, DISCUSSION}

\section{Total proteins}

The levels of total protein in the blood serum in Rhenish, Italian and Landes breeds of geese during the experimental period are presented in table $\mathrm{I}$. study.

The total protein values did not show a definite trend during the period of the

Comparing the different breeds of geese with each other, the protein levels showed slight fluctuations from one breed to another at the different sampling periods. The analysis of variance indicated that the difference between the breeds in total protein level was significant at the first and fourth week of force feeding (tables 2 and 3).

\section{Serum protein fractions}

\section{a) Albumin fraction.}

The percentages of albumin to the total serum protein before and during the force feeding period in the breeds of geese studied are shown in table 4 .

The albumin percentages in Rhenish, Italian and Landes breeds increased rapidly at the first week of force feeding, then generally showed a gradual increase 


\section{TABLE I}

Total protein level in the blood serum (g/10o ml), before and during force feeding

Protéines sériques totales $(\mathrm{g} / \mathrm{1} 00 \mathrm{ml})$ avant et pendant le gavage

\begin{tabular}{|c|c|c|c|c|c|c|c|c|c|c|}
\hline $\begin{array}{c}\text { Breeds } \\
\text { Race }\end{array}$ & \multicolumn{3}{|c|}{$\begin{array}{l}\text { Rhenish } \\
\text { Rhenane }\end{array}$} & \multicolumn{3}{|c|}{$\begin{array}{l}\text { Italian } \\
\text { Italienne }\end{array}$} & \multicolumn{3}{|c|}{$\begin{array}{l}\text { Landes } \\
\text { Landaise }\end{array}$} & \multirow{2}{*}{$\begin{array}{c}\text { Total } \\
\text { Average }\end{array}$} \\
\hline Intervals & $n$ & $\overline{\mathbf{x}}$ & $\mathrm{CV} \%$ & $n$ & $\overline{\mathbf{X}}$ & $\mathrm{CV} \%$ & $n$ & $\overline{\mathbf{X}}$ & $\mathrm{CV} \%$ & \\
\hline $\begin{array}{l}\text { Before force feeding } \\
\text { Avant gavage }\end{array}$ & 13 & 4.69 & 26.2 & 13 & 4.38 & 10.8 & 13 & 4.29 & 7.9 & 4.45 \\
\hline $\begin{array}{l}\text { After one week } \\
\text { Après } 1 \text { semaine }\end{array}$ & 13 & 4.47 & 6.6 & 13 & 4.25 & 11.4 & 13 & 5.07 & 14.0 & 4.59 \\
\hline $\begin{array}{l}\text { After two weeks } \\
\text { Après } 2 \text { semaines }\end{array}$ & 13 & 4.12 & 10.2 & 13 & 4.10 & 10.1 & 13 & 4.04 & 5.7 & 4.08 \\
\hline $\begin{array}{l}\text { After three weeks } \\
\text { Après } 3 \text { semaines }\end{array}$ & 13 & 4.38 & 11.8 & 13 & 4.33 & 12.5 & 13 & 4.34 & 8.5 & 4.35 \\
\hline $\begin{array}{l}\text { After four weeks } \\
\text { Après } 4 \text { semaines }\end{array}$ & 13 & 4.41 & 13.7 & 13 & 4.07 & 11.3 & 13 & 4.61 & 9.1 & 4.36 \\
\hline
\end{tabular}

\section{TABLE 2}

Test of significance (" $\mathrm{F}$ " value for the significant cases only) of breed in total serum proteins

Effet de la race (valeur de a $\mathrm{F}$ " pour les cas significatifs seulement) sur les protéines sériques totales

\begin{tabular}{|c|c|}
\hline Items & " $F$ "value \\
\hline $\begin{array}{l}\text { First week of force feeding } \\
\text { Ire semaine de gavage }\end{array}$ & $8.9231 *$ \\
\hline $\begin{array}{l}\text { Fourth week of force feeding } \\
4^{e} \text { semaine de gavage }\end{array}$ & $4.000 * *$ \\
\hline
\end{tabular}

* $\mathbf{P}<.01$

$* * \mathrm{P}<.05$ 


\section{TABLE 3}

The values of least significant differences (L.S.D.) for the total protein

Valeurs des plus petites différences significatives (L.S.D.) pour les protéines totales

\begin{tabular}{|c|c|c|c|}
\hline & $\begin{array}{l}\text { Rhenish } \\
\text { and Italian } \\
\text { Rhénane } \\
\text { et Italienne }\end{array}$ & $\begin{array}{c}\text { Rhenish } \\
\text { and Landes } \\
\text { Rhénane } \\
\text { et Landaise }\end{array}$ & $\begin{array}{l}\text { Landes } \\
\text { and Italian } \\
\text { Landaise } \\
\text { et Italienne }\end{array}$ \\
\hline $\begin{array}{l}\text { First week of force feeding } \\
1^{\text {re }} \text { semaine de gavage }\end{array}$ & $0.22 *$ & $0.60^{*}$ & $0.82 *$ \\
\hline $\begin{array}{c}\text { Fourth week of force feeding } \\
4^{\mathrm{e}} \text { semaine de gavage }\end{array}$ & $0.34^{*}$ & 0.20 & $0.54 *$ \\
\hline$* \mathrm{P}<.01$ & & & \\
\hline
\end{tabular}

TABLE 4

Percentage of albumin to total serum protein

Pourcentage d'albumine dans les protéines sériques totales

\begin{tabular}{|c|c|c|c|c|c|c|c|c|c|c|}
\hline \multirow{2}{*}{$\begin{array}{c}\begin{array}{c}\text { Breeds } \\
\text { Race }\end{array} \\
\text { Intervals }\end{array}$} & \multicolumn{3}{|c|}{$\begin{array}{l}\text { Rhenish } \\
\text { Rhenane }\end{array}$} & \multicolumn{3}{|c|}{$\begin{array}{l}\text { Italian } \\
\text { Italienne }\end{array}$} & \multicolumn{3}{|c|}{$\begin{array}{l}\text { Landes } \\
\text { Landaise }\end{array}$} & \multirow{2}{*}{$\begin{array}{l}\text { Total } \\
\text { Average }\end{array}$} \\
\hline & $n$ & $\overline{\mathrm{x}}$ & $\mathrm{CV} \%$ & $n$ & $\overline{\mathrm{x}}$ & $\mathrm{CV} \%$ & $n$ & $\overline{\mathrm{x}}$ & $\mathrm{CV} \%$ & \\
\hline $\begin{array}{l}\text { Before force feeding } \\
\text { Avant gavage }\end{array}$ & 13 & 42.0 & 11.1 & 13 & 44.7 & 16.7 & 13 & 43.3 & 13.8 & 43.3 \\
\hline $\begin{array}{l}\text { After one week } \\
\text { Apres } 1 \text { semaine }\end{array}$ & 13 & 47.8 & 18.9 & 13 & 48.4 & 16.6 & 13 & 44.1 & 22.9 & 46.7 \\
\hline $\begin{array}{l}\text { After two weeks } \\
\text { Après } 2 \text { semaines }\end{array}$ & 13 & 48.7 & 20.9 & 13 & 48.6 & 9.5 & 13 & 47.3 & 11.1 & 48.2 \\
\hline $\begin{array}{l}\text { After three weeks } \\
\text { Après } 3 \text { semaines }\end{array}$ & 13 & 49.8 & 12.0 & 13 & 50.3 & 14.4 & 13 & 44.7 & 19.1 & 48.3 \\
\hline $\begin{array}{l}\text { After four weeks } \\
\text { Après } 4 \text { semaines }\end{array}$ & 13 & 52.5 & 19.5 & 13 & 50.5 & 15.7 & 13 & 51.4 & 13.6 & 51.5 \\
\hline
\end{tabular}


until the end of the experiment (table 4). The reason for such increase was not clear. Studying the efficiency of the liver function during the course of force feeding may throw more light on the previous results.

Comparing the breeds of geese studied, it was observed that the albumin percentage was higher in the Italian breed than in the other breeds in most of the ages studied (table 4). However, the statistical analysis showed no significant differences between these breeds either before or during the force feeding period (table 2 ).

\section{b) Globulin fraction.}

The total globulin percentage decreased generally during the period of the study until it became lower than fifty per cent at the end (table 5). From another viewpoint, the percentage of globulin was higher than that of albumin in the geese blood before cramming (table 5). This agrees with the findings of WOLFE et al. (I957) in fowls.

The total globulin percentages according to breed showed no definite trend (table 5). The breed differences were not significant (table 2).

TABLE 5

Percentage of total globulin in the blood sera

Pourcentage de globulines dans le sérum

\begin{tabular}{|c|c|c|c|c|c|c|c|c|c|c|}
\hline \multirow{2}{*}{$\begin{array}{c}\begin{array}{c}\text { Breeds } \\
\text { Race }\end{array} \\
\text { Intervals }\end{array}$} & \multicolumn{3}{|c|}{$\begin{array}{l}\text { Rhenish } \\
\text { Rhénane }\end{array}$} & \multicolumn{3}{|c|}{$\begin{array}{l}\text { Italian } \\
\text { Italienne }\end{array}$} & \multicolumn{3}{|c|}{$\begin{array}{l}\text { Landes } \\
\text { Landaise }\end{array}$} & \multirow{2}{*}{$\begin{array}{c}\text { Total } \\
\text { Average }\end{array}$} \\
\hline & $n$ & $\bar{x}$ & $\mathrm{CV} \%$ & $n$ & $\overline{\mathbf{x}}$ & $\mathrm{CV} \%$ & $n$ & $\overline{\mathrm{x}}$ & $\mathrm{CV} \%$ & \\
\hline $\begin{array}{l}\text { Before force feeding } \\
\text { Avant gavage }\end{array}$ & 13 & 58.0 & 8.1 & 13 & 55.4 & 13.4 & 13 & 56.7 & 9.3 & 56.7 \\
\hline $\begin{array}{l}\text { After one week } \\
\text { Après } 1 \text { semaine }\end{array}$ & 13 & 51.7 & 17.7 & 13 & 51.7 & 15.2 & 13 & 56.0 & 17.8 & 53.1 \\
\hline $\begin{array}{l}\text { After two weeks } \\
\text { Après } 2 \text { semaines }\end{array}$ & 13 & 49.7 & 17.1 & 13 & 51.8 & 9.5 & 13 & 52.3 & 9.9 & 51.3 \\
\hline $\begin{array}{l}\text { After three weeks } \\
\text { Après } 3 \text { semaines }\end{array}$ & 13 & 50.2 & 11.6 & 13 & 49.7 & 14.5 & 13 & 55.3 & 15.4 & 51.7 \\
\hline $\begin{array}{l}\text { After four weeks } \\
\text { Après } 4 \text { semaines }\end{array}$ & 13 & 47.5 & 21.6 & 13 & 49.5 & 14.6 & 13 & 48.9 & 15.0 & 48.7 \\
\hline
\end{tabular}

c) The ratio of albumin to globulin $(A / G$ ratio).

The albumin to globulin ratio in the blood serum of geese was 0.78 before cramming (table 6). It increased until it became I.I4 at the end of the experiment. This increase may be a result of force feeding carbohydrates.

When classifying the above-mentioned ratio according to breed, there was no definite trend (table 6). The breed differences were not significant (table 2). 
TABLE 6

The albumin to globulin ratio in the blood serum

Rapport albumine/globulines dans le sérum

\begin{tabular}{|c|c|c|c|c|c|c|c|c|c|c|}
\hline \multirow{2}{*}{$\begin{array}{c}\begin{array}{c}\text { Breeds } \\
\text { Race }\end{array} \\
\text { Intervals }\end{array}$} & \multicolumn{3}{|c|}{$\begin{array}{l}\text { Rhenish } \\
\text { Rhenane }\end{array}$} & \multicolumn{3}{|c|}{$\begin{array}{l}\text { Italian } \\
\text { Italienne }\end{array}$} & \multicolumn{3}{|c|}{$\begin{array}{l}\text { Landes } \\
\text { Landaise }\end{array}$} & \multirow{2}{*}{$\begin{array}{l}\text { Total } \\
\text { Average }\end{array}$} \\
\hline & $n$ & $\overline{\mathbf{x}}$ & $\mathrm{CV} \%$ & $n$ & $\overline{\mathrm{x}}$ & $\mathrm{CV} \%$ & $n$ & $\overline{\mathrm{x}}$ & $\mathrm{CN} \%$ & \\
\hline $\begin{array}{l}\text { Before force feeding } \\
\text { Avant gavage }\end{array}$ & 13 & 0.73 & 21.6 & 13 & 0.84 & 29.8 & 13 & 0.78 & 22.6 & 0.78 \\
\hline $\begin{array}{l}\text { After one week } \\
\text { Après } 1 \text { semaine }\end{array}$ & 13 & 0.96 & 35.2 & 13 & 0.97 & 30.0 & 13 & 0.85 & 36.3 & 0.93 \\
\hline $\begin{array}{l}\text { After two weeks } \\
\text { Apres } 2 \text { semaines }\end{array}$ & 13 & 1.06 & 31.4 & 13 & 0.96 & 18.5 & 13 & 0.92 & 22.3 & 0.98 \\
\hline $\begin{array}{l}\text { After three weeks } \\
\text { Après } 3 \text { semaines }\end{array}$ & 13 & 1.02 & 27.5 & 13 & 1.04 & 30.1 & 13 & 0.85 & 41.8 & 0.97 \\
\hline $\begin{array}{l}\text { After four weeks } \\
\text { Après } 4 \text { semaines }\end{array}$ & 13 & 1.23 & 44.0 & 13 & 1.08 & 32.3 & 13 & 1.10 & 32.8 & 1.14 \\
\hline
\end{tabular}

TABLE 7

Total lipid of blood serum before and during force feeding $(\mathrm{mg} / 100 \mathrm{ml})$

Lipides sériques totaux $(\mathrm{mg} / 100 \mathrm{ml})$ avant et pendant le gavage

\begin{tabular}{|c|c|c|c|c|c|c|c|c|c|c|}
\hline \multirow{2}{*}{$\begin{array}{c}\begin{array}{c}\text { Breeds } \\
\text { Race }\end{array} \\
\text { Intervals }\end{array}$} & \multicolumn{3}{|c|}{$\begin{array}{l}\text { Rhenish } \\
\text { Rhenane }\end{array}$} & \multicolumn{3}{|c|}{$\begin{array}{l}\text { Italian } \\
\text { Italienne }\end{array}$} & \multicolumn{3}{|c|}{$\begin{array}{l}\text { Landes } \\
\text { Landaise }\end{array}$} & \multirow{2}{*}{$\begin{array}{l}\text { Total } \\
\text { Average }\end{array}$} \\
\hline & $n$ & $\overline{\mathrm{x}}$ & $\mathrm{CV} \%$ & $n$ & $\bar{x}$ & $\mathrm{CV} \%$ & $n$ & $\bar{x}$ & $\mathrm{CV} \%$ & \\
\hline $\begin{array}{l}\text { Before force feeding } \\
\text { Avant gavage }\end{array}$ & 13 & 725.2 & 29.1 & 13 & 775.2 & 22.0 & 13 & 777.5 & 21.3 & 759.3 \\
\hline $\begin{array}{l}\text { After one week } \\
\text { Après } 1 \text { semaine }\end{array}$ & 12 & 1350.0 & 28.3 & 13 & 1323.1 & 24.3 & 13 & 1335.7 & 21.3 & 1335.9 \\
\hline $\begin{array}{l}\text { After two weeks } \\
\text { Après } 2 \text { semaines }\end{array}$ & 12 & 1499.3 & 14.6 & 13 & 1488.7 & 20.9 & 13 & 1673.3 & 12.4 & 1555.2 \\
\hline $\begin{array}{l}\text { After three weeks } \\
\text { Apres } 3 \text { semaines }\end{array}$ & 13 & 1 856.9 & 18.7 & 13 & 2017.5 & 26.5 & 13 & 1748.3 & 29.6 & 1874.2 \\
\hline $\begin{array}{l}\text { After four weeks } \\
\text { Après } 4 \text { semaines }\end{array}$ & 13 & 2362.5 & 19.3 & 13 & 2284.9 & 32.3 & 13 & 2143.4 & 20.8 & 2271.3 \\
\hline
\end{tabular}




\section{Total lipid}

The total lipid of the blood serum showed a rapid increase after the beginning of force feeding in all the breeds of geese (table 7 ). The average value of total lipid in all the breeds studied was $759.3 \mathrm{mg} / \mathrm{roo} \mathrm{ml}$ before force feeding. It then increased successively until it became $227 \mathrm{r} .3 \mathrm{mg} / \mathrm{I} 00 \mathrm{ml}$ at the end of the experiment (table 7 ).

When comparing the breeds of geese, the total lipid values did not show a definite trend during the study (table 7 ). Statistically, the breed differences in serum lipids were not significant.

\section{Cholesterol}

The values of the total average of the sera cholesterol increased gradually upto the end of the last week of force feeding in all the breeds of geese as shown in

\section{TABLE 8}

Serum cholesterol before and during force feeding period $(\mathrm{mg} / \mathrm{100} \mathrm{ml})$ Cholestérol sérique $(\mathrm{mg} / \mathrm{roo} \mathrm{ml})$ avant et pendant le gavage

\begin{tabular}{|c|c|c|c|c|c|c|c|c|c|c|}
\hline $\begin{array}{l}\text { Breeds } \\
\text { Race }\end{array}$ & \multicolumn{3}{|c|}{$\begin{array}{l}\text { Rhenish } \\
\text { Rhenane }\end{array}$} & \multicolumn{3}{|c|}{$\begin{array}{l}\text { Italian } \\
\text { Italienne }\end{array}$} & \multicolumn{3}{|c|}{$\begin{array}{l}\text { Landes } \\
\text { Landaise }\end{array}$} & \multirow{2}{*}{$\begin{array}{l}\text { Total } \\
\text { Average }\end{array}$} \\
\hline Intervals & $n$ & $\overline{\mathbf{x}}$ & CV \% & $n$ & $\overline{\mathbf{x}}$ & $\mathrm{CV} \%$ & $n$ & $\bar{x}$ & CV \% & \\
\hline $\begin{array}{c}\text { Before force feeding } \\
\text { Avant gavage }\end{array}$ & 13 & 128.5 & 17.5 & 13 & 148.5 & 26.5 & 13 & 135.8 & 24.1 & 137.6 \\
\hline $\begin{array}{l}\text { After one week } \\
\text { Après } 1 \text { semaine }\end{array}$ & 12 & 145.9 & 27.3 & 13 & 124.2 & 22.2 & 13 & 148.1 & 44.4 & 138.7 \\
\hline $\begin{array}{l}\text { After two weeks } \\
\text { Après } 2 \text { semaines }\end{array}$ & 12 & 137.6 & 22.9 & 13 & 156.5 & 26.1 & 13 & 130.8 & 13.5 & 141.7 \\
\hline $\begin{array}{l}\text { After three weeks } \\
\text { Apres } 3 \text { semaines }\end{array}$ & 13 & 150.4 & 15.9 & 13 & 180.4 & 14.1 & 13 & 140.8 & 17.0 & 157.2 \\
\hline $\begin{array}{l}\text { After four weeks } \\
\text { Après } 4 \text { semaines }\end{array}$ & 13 & 157.7 & 17.5 & 13 & 244.2 & 48.5 & 13 & 165.0 & 17.4 & 189.0 \\
\hline
\end{tabular}

TABI,E 9

Test of significance (" $\mathrm{F}$ " value for the significant cases only) of breed in sera cholesterol level Effet de la race (" $\mathrm{F}$ " pour les cas significatifs) sur le cholestérol sérique

\begin{tabular}{|c|c|}
\hline Items & “F” value \\
\hline $\begin{array}{c}\text { Third week of force feeding } \\
\text { 3e semaine de gavage }\end{array}$ & $9.3926 *$ \\
\hline $\begin{array}{c}\text { Fourth week of force feeding } \\
4^{\mathrm{e}} \text { semaine de gavage }\end{array}$ & $5.6646^{*}$ \\
\hline
\end{tabular}

$* \mathrm{P}<0.01$ 
table 8. These values were I37.6, I38.7, I4I.7, I57.2 and I89.0 $\mathrm{mg} / \mathrm{Ioo} \mathrm{m} 1$ at the successive sampling times, respectively.

The breed showed a highly significant effect on the amount of the cholestrol at the third and fourth weeks of force feeding (tables 9 and Io).

\section{TABLE IO}

The values of least significant differences (L.S.D.) for the cholesterol means

Differences between breeds (mg/ roo $\mathrm{ml}$ )

Valeurs des plus petites différences significatives (L.S.D.) pour le cholestérol

Différences entre races $(\mathrm{mg} / \mathrm{Ioo} \mathrm{ml})$

\begin{tabular}{|c|c|c|c|}
\hline & $\begin{array}{l}\text { Rhenish } \\
\text { and Italian } \\
\text { Rhénane } \\
\text { et Italienne }\end{array}$ & $\begin{array}{l}\text { Rhenish } \\
\text { and Landes } \\
\text { Rhénane } \\
\text { et Landaise }\end{array}$ & $\begin{array}{l}\text { Landes } \\
\text { and Italian } \\
\text { Landaise } \\
\text { et Italienne }\end{array}$ \\
\hline 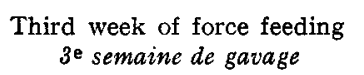 & $30.0^{*}$ & $9.6 *$ & $39.6 * *$ \\
\hline $\begin{array}{l}\text { Fourth week of force feeding } \\
4^{\mathrm{e}} \text { semaine de gavage }\end{array}$ & $13.5 *$ & 7.3 & $20.8^{* *}$ \\
\hline $\begin{aligned} * \mathrm{P} & <.05 \\
* * \mathrm{P} & <.01\end{aligned}$ & & & \\
\hline
\end{tabular}

Rę̧u pour publication en octobre 1972.

\section{ACKNOWLEDGEMENT}

The authors are grateful to Pr Dr. A. MAGYARI, Chairman of the Department of Animal Production, University of Agricultural Sciences, Gödöllö, Hungary, for hisk ind help during the course of the study.

\section{RÉSUMÉ}

\section{ÉVOLUTION DU CHOLESTÉROL, DES LIPIDES ET DES PROTÉINES SÉRIQUES} AU COURS DU GAVAGE CHEZ L'OIE

Dix femelles et dix mâles de chacune des trois races Rhénane, Italienne et Landaise, sont gavés pendant quatre semaines. Des prises de sang hebdomadaires, commencées la semaine qui précède le gavage, permettent de suivre l'évolution dans le sérum des protéines totales et de leurs fractions (albumine, globulines), des lipides totaux et du cholestérol.

Les protéines totales n'évoluent pas beaucoup au cours de l'expérience. Les différences entre races ne sont significatives que la quatrième semaine du gavage. Plus précisément, la différence est hautement significative entre la race Rhénane et la race Italienne et entre cette dernière et la race Landaise. 
Pour les trois races, le pourcentage d'albumine augmente au cours du gavage, tandis que le pourcentage de globulines diminue. Le rapport albumine/globulines passe de 0,78 avant le gavage à I, I 4 à la fin de l'expérience. Il n'y a pas de différences significatives dues à la race pour les pourcentages d'albumine et de globulines et le rapport albumine/globuline.

Les lipides sériques et le cholestérol augmentent au cours du gavage. Pour ces deux paramètres les différences entre races ne sont pas significatives avant le gavage, mais la race a un effet hautement significatif sur le taux de cholestérol au cours de la troisième et de la quatrième semaine de gavage.

\section{RÉFÉRENCES BIBLIOGRAPHIQUES}

Balint P., 1962. Klinikai laboratoriumi diagnostika, Medicina Kiado-harmadik, bovigett es atdolgozott kiadas. Budapest, Hungary.

Blum J.-C., Gaumeton J.-C., Mur J.-P., Leclerce B., I97o. Modifications de la valeur des normes sanguines en fonction du degré de stéatose hépatique chez l'oie gavée. Ann. Rech. Véter., 1 , I67-r 78 .

Dittmer V. A., Anno., r965. Plasmaei weiss und elektrophorese. 3. Papeilektrophorese als Routinemethode. Veb. Gustav Fisher Verlag Jema, 77-I34.

Forenbacher S., I969. Changements dans la composition du foie et du sang des oies au cours du gavage. Journées de l'Oie, 57, 4-5-6: 136.

LEVINE J., ZAK B., I969. Quantitative determination of total cholesterol in serum by the automatic colorimetric procedure. Clin. Chem. Acta, 10, 38I-384.

Svab J., I967. Biometriai modszerek a mezogazdasagi kutatasban. Mezogazdasagi Kiado. Budapest, Hungary.

Tournut J., Montlaur-Ferradou P., I969. Normes biochimiques sanguines au cours du gavage, Journees de l'Oie, 57, 4-5-6: 137 .

Wolfe H. R., Meuller A., Neess J., Temphis C., 1957. Precipitin production in chickens. I6. The relationship of age to antibody production. J. Immunol., 79, I42.

WYRZYLOWSKI Z., BIELINSKI K., Ig6g. Les changements de la formule sanguine au cours du gavage. Journee de l'Oie, $57,4-5-6:$ : 36 . 